\title{
Incidence of acute kidney disease after receiving hematopoietic stem cell transplantation: a single-center retrospective study
}

\author{
Akira Mima ${ }^{\text {Corresp.. }}{ }^{1}$, Kousuke Tansho $^{1}{ }^{1}$, Dai Nagahara ${ }^{1}$, Kazuo Tsubaki ${ }^{2}$ \\ 1 Department of Nephrology, Kindai University Faculty of Medicine, Kindai University Nara Hospital, Nara, Japan \\ 2 Department of Hematology, Kindai University Faculty of Medicine, Kindai University Nara Hospital, Nara, Japan \\ Corresponding Author: Akira Mima \\ Email address: amima@med.kindai.ac.jp
}

Background. Previous reports have shown that acute kidney injury (AKI) is common after hematopoietic stem cell transplantation (HSCT), which is a crucial treatment for patients with hematological disorders. AKI could increase mortality and induce adverse effects including the development of chronic kidney disease. The incidence of AKI in association with HSCT reportedly varies significantly because several definitions of AKI have been adopted. Acute kidney disease (AKD) is a new concept that can clinically define both AKI and persistent decreases in glomerular filtration rate (GFR) state. We conducted a retrospective cohort study to determine the incidence of AKD after HSCT. Methods. This study included 108 patients aged between 16 and 70 years undergoing HSCT. In this study, AKD included clinical condition of AKI or subacute decreases in GFR. AKI was defined according to the Kidney Disease: Improving Global Outcomes guidelines based on serum creatinine. However, urine output data were not included to define AKI because the database lacked some of these data. Comparisons were made between groups using the Mann-Whitney U test. Results. AKD occurred in 17 patients (15.7\%). There were significant differences between the AKD and non-AKD with respect to ABO-incompatible HSCT ( $p=0.001$ ) and incidence of acute graft versus host disease (GVHD) after HSCT $(p<0.001)$. The 100-day overall survival of patients with AKD and without AKD after HSCT was $70.6 \%$ and $79.8 \%$, respectively $(p=0.409)$. Discussion. ABO-incompatible HSCT and acute GVHD after HSCT were risk factors for the incidence of AKD. However, we could not find a significant association between AKD after HSCT and mortality. 


\section{Incidence of acute kidney disease after receiving}

\section{2 hematopoietic stem cell transplantation: a single-}

\section{3 center retrospective study}

4

Akira Mima $^{1 *}$, Kousuke Tansho ${ }^{1}$, Dai Nagahara ${ }^{1}$, and Kazuo Tsubaki ${ }^{2}$

${ }^{1}$ Department of Nephrology, Kindai University Nara Hospital, Kindai University Faculty of Medicine, Nara, Japan

${ }^{2}$ Department of Hematology, Kindai University Nara Hospital, Kindai University Faculty of Medicine, Nara, Japan

${ }^{*}$ Corresponding author: Akira Mima, M.D., Ph.D.

Department of Nephrology, Kindai University Nara Hospital

Kindai University Faculty of Medicine, 1248-1 Otoda-cho, Ikoma-city, Nara, 630-0293, Japan Tel: 81-743-77-0880, Fax: 81-743-77-0890 E-mail: amima@med.kindai.ac.jp

\section{ABSTRACT}

Background. Previous reports have shown that acute kidney injury (AKI) is common after hematopoietic stem cell transplantation (HSCT), which is a crucial treatment for patients with hematological disorders. AKI could increase mortality and induce adverse effects including the development of chronic kidney disease. The incidence of AKI in association with HSCT reportedly varies significantly because several definitions of AKI have been adopted. Acute kidney disease (AKD) is a new concept that can clinically define both AKI and persistent decreases in glomerular filtration rate (GFR) state. We conducted a retrospective cohort study to determine the incidence of AKD after HSCT.

Methods. This study included 108 patients aged between 16 and 70 years undergoing HSCT. In this study, AKD included clinical condition of AKI or subacute decreases in GFR. AKI was defined according to the Kidney Disease: Improving Global Outcomes guidelines based on serum creatinine. However, urine output data were not included to define AKI because the 
30 database lacked some of these data. comparisons were made between groups using the Mann-

31 Whitney U test.

32 Results. AKD occurred in 17 patients (15.7\%). There were significant differences between the $\mathrm{AKD}$ and non-AKD with respect to ABO-incompatible HSCT $(\mathrm{p}=0.001)$ and incidence of acute graft versus host disease (GVHD) after HSCT $(\mathrm{p}<0.001)$. The 100-day overall survival of patients with AKD and without AKD after HSCT was $70.6 \%$ and $79.8 \%$, respectively $(\mathrm{p}=0.409)$.

Discussion. ABO-incompatible HSCT and acute GVHD after HSCT were risk factors for the incidence of AKD. However, we could not find a significant association between AKD after HSCT and mortality.

\section{INTRODUCTION}

Hematopoietic stem cell transplantation (HSCT) is a fundamental therapy for high risk and refractory hematological diseases. Several types of HSCT procedures exist based on conditioning therapy (myeloablative or reduced intensity), donor source (autologous and allogenic, including matched related, matched unrelated, haploidentical and umbilical cord) and stem cell source (peripheral blood stem cells or bone marrow stem cells) [1,2]. Acute kidney disease $(\mathrm{AKD})$ can lead to chronic kidney disease (CKD) with increased all-cause mortality. Although acute kidney injury (AKI) is a well-defined concept, AKD definition is relatively new [3]. To diagnose AKI more accurately, the new concept of AKI definition has been proposed by the Kidney Disease Improving Global Outcomes (KDIGO), which is based on and modified by the risk, injury, failure, loss of function, end-stage renal disease (RIFLE) classification and the Acute Kidney Injury Network (AKIN) criteria [3-6]. AKD is defined as subacute reduction in glomerular filtration rate (GFR), including AKI clinical states [7, 8].

Previous reports have shown that AKI is relatively common after HSCT, and it could increase mortality and induce adverse effects including the development of CKD [9]. Previous reports indicated that the incidence of AKI in association with HSCT ranged from 20\% to $73 \%$ [10], while other studies indicated $42 \%$ to $92 \%$ [11]. Study results based on these data have been varied because several definitions of AKI have been adopted.

Acute graft versus host disease (GVHD) occurs within the first 3 months after HSCT and involves the reactivity of donor immune cells against host tissues [12]. Therefore, when evaluating kidney damage in acute GVHD, the conventional definition of AKI, in which AKI 
61 should be diagnosed within 1 week, may not be suitable. Thus, we used the definition of AKD, 62 which is a new concept and could clinically define both AKI and persistent decreases in GFR 63 state. We conducted a retrospective cohort study to determine the incidence of AKD after HSCT. 64

65 MATERIALS \& METHODS

66

\section{Patient groups}

All procedures performed in studies involving human participants were in accordance with the ethical standards of the institutional and/or national research committee and with the 1964 Helsinki declaration and its later amendments or comparable ethical standards. This is a retrospective medical record review study. The retrospective waiver of consent was obtained from the clinical study ethics review board of Kindai University Nara Hospital (approval number: 18-6).

This study included a retrospective cohort of 108 patients who underwent HSCT at Kindai University Nara Hospital from May 2006 to April 2016. Data were collected and analyzed retrospectively using electronic medical records maintained by the Department of Nephrology and Hematology at Kindai University Nara Hospital. Data including year of HSCT, HSCT characteristics (e.g., underlying disease, donor type, total body irradiation, ABO incompatibility, and calcineurin inhibitor-based GVHD prophylaxis), indications for HSCT, acute GVHD, AKD, and survival outcomes were obtained from the electronic medical records and transplant database.

\section{Definition of AKD}

AKD was defined to include clinical condition of AKI or subacute decreases in GFR (i.e., GFR $<60 \mathrm{ml} / \mathrm{min} / 1.73 \mathrm{~m}^{2}$ for less than 3 months or decrease in GFR by $\geq 35 \%$ or increase in serum creatinine by $>50 \%$ for less than 3 months) [7, 8]. AKI was defined according to KDIGO guidelines based on serum creatinine [3]. However, urine output data were not included in our definition of AKI because some data were not available in the database.

\section{Statistical analysis}

Continuous variables were presented as medians with interquartile ranges (IQRs). 
92 Comparisons were made between groups using the Mann-Whitney U test; categorical variables

93 were presented as numbers (percentage) and compared using the Fisher's exact tests. According

94 to clinical features and previous reports which indicated the association with AKD after HSCT,

9512 variables (age, sex, height, weight, underlying disease, with or without hypertension, with or

96 without diabetes mellitus, total body irradiation, ABO compatibility, acute GVHD, calcineurin

97 inhibitor-based GVHD prophylaxis, and donor and cell source) were eligible for this study.

98 The 100-day overall survival of patients with or without AKD after HSCT was

99 estimated using the Kaplan-Meier method and compared using the log-rank test. All analysis was

100 performed using StatView (SAS Institute, Cary, CA, USA). Statistical significance was defined

101 as $\mathrm{p}<0.05$.

102

\section{RESULTS}

We determined the clinical characteristics of 108 patients who underwent HSCT. The median age of the study participants was 49 (IQRs; 16-70) years, and 39 (36.1\%) were women. The median height was 166 (IQRs; 149-184) cm and body weight was 58 (IQRs; 35-95) kg. Underlying diseases were as follows: acute lymphoblastic leukemia (ALL: 15 cases, 13.9\%), acute myeloblastic leukemia (AML: 43 cases, 39.8\%), chronic myeloblastic leukemia (CML: 4 cases, 3.7\%), myelodysplastic syndromes (MDS: 10 cases, 9.3\%), multiple myeloma (MM: 5 cases, 4.6\%), aplastic anemia (AA: 6 cases, 5.6\%), and others (25 cases, 23.1\%). Hypertension

111 and diabetes were observed in $12(11.1 \%)$ and 9 cases $(8.3 \%)$, respectively. Body irradiation

112 more than 8 Gy was observed in 79 cases (73.1\%). ABO-incompatible HSCT was performed in

11318 cases (16.7\%). Acute GVHD after HSCT occurred in 17 cases (15.7\%) and calcineurin-based

114 GVHD prophylaxis was given in 67 cases (62.0\%). Donor and cell sources were as follows:

115 allogenic bone marrow transplantation (Allo-BMT: 20 cases, 18.5\%), unrelated umbilical cord

116 blood transplantation (UR-CBT: 38 cases, 35.2\%), unrelated bone marrow transplantation (UR-

117 BT: 26 cases, 24.1\%), allogenic peripheral blood stem cell transplantation (Allo-PBSCT: 11

118 cases, 10.2\%), and autologous peripheral blood stem cell transplantation (Auto-PBSCT: 16 cases,

119 14.8\%). AKD developed in 17 cases (15.7\%) within 100 days of HSCT. Furthermore, four out of

12017 AKD cases required hemodialysis. We assessed the association of AKD with the clinical

121 characteristics as mentioned above. There were significant differences between the AKD and

122 non-AKD with respect to ABO-incompatible HSCT $(p=0.001)$ and incidence of acute GVHD 
123 after HSCT $(\mathrm{p}<0.001)$. However, we could not find a significant association between AKD and

124 other clinical characteristics (Table 1). Then, we analyzed the 100-day overall survival of

125 patients with AKD and without AKD after HSCT. The overall survival rate was $70.6 \%$ and

$12679.8 \%$, respectively ( $\mathrm{p}=0.409)$. Thus, we could not find a significant association between HSCT-

127 induced AKD and mortality (Figure 1).

128

\section{DISCUSSION}

This retrospective, single-center study focused on the incidence of AKD after HSCT.

131 Our results could offer the probability of searching the risk and prognostic factor of AKD after

132 HSCT and provide a deeper understanding of this disorder. We found that the incidence of AKD

133 after HSCT was 15.7\%. Furthermore, ABO-incompatible HSCT and the incidence of acute

134 GVHD were independently associated with AKD. However, we could not find a significant

135 association between AKD and mortality after HSCT.

136 Previous reports indicated that the incidence of AKI among HSCT recipients varies

137 widely from $20 \%$ to $92 \%[10,11]$. This difference has varied significantly because of the

138 variations in AKI definitions. Especially, most previous studies defined AKI using simply the

139 doubling of serum creatinine, and this could exclude early-stage AKI. KDIGO proposed a new

140 AKI definition which has been integrated from AKIN and RIFLE [3]. However, despite using

141 the KDIGO definition, acute GVHD-related kidney injury could not be evaluated properly,

142 because most acute GVHDs occur within 100 days after HSCT. There were no accurate urinary

143 volume data in our study, but when evaluating AKI according to serum creatinine level alone,

144 only two cases (stages 1 and 3) could be diagnosed as AKI. These results also support that the conventional AKI definition could not be suitable for acute GVHD-related renal injury.

146 Incidence of AKD in our study was lower than previous reports. Several reasons could

147 be considered. First, medications that can decrease glomerular perfusion, such as renin

148 angiotensin system inhibitors (RASI) or NSAIDs were used in our study very few; RASI were

149 used in 2 cases (1.9\%) and NSAIDs were used in 4 cases (3.7\%), respectively. Second, diabetes

150 which could exacerbate renal function was recognized only 9 cases $(8.3 \%)$ in our study. Third, as

151 previously indicated, early involvement of the nephrologist in our case might prevent incidence

152 of AKD $[10,13]$.

153 Induction of combination therapy cyclosporine or tacrolimus, calcineurin inhibitors 
154 (CNIs) and methotrexate could be superior to calcineurin inhibitors alone in the prophylaxis of 155 acute GVHD. Although there was no correlation between the incidence of AKD and the use of 156 calcineurin inhibitors (CNIs) in our study, several mechanisms by which cyclosporine use leads 157 to renal injury are proposed. Previous reports indicated that CNIs induced glomerular endothelial 158 dysfunction and renal artery smooth muscle cell contraction, leading to AKD [13, 14]. Our study 159 showed no correlation between CNIs and incidence of AKD. Much of the renal toxicity of CNIs 160 is thought to be dose dependent. One possibility is that CNIs levels in the blood were monitored 161 strictly to prevent side effects in our study. CNIs could activate renin angiotensin II system 162 (RAS), which increases phospho-c-Raf (Ser338) and Erk1/2 phosphorylation increasing 163 extracellular matrix [15]. RAS also causes vasoconstriction of renal arterioles, inducing AKI [14, $16416,17]$.

The association between AKI and the incidence of GVHD has been reported. After HSCT, kidneys are exposed to GVHD-induced systemic inflammation. Donor T-cells and endothelial dysfunction are also key aspects of developing AKI in GVHD [13]. Further, increases in several cytokines could trigger kidney injury in acute GVHD; when acute GVHD occurred, interleukin (IL)-2, IL-6, or tumor necrosis factor- $\alpha$ was upregulated in the kidney [1820]. These cytokines are produced during the initial step of GVHD, resulting in AKD. Furthermore, prolonged increases in these inflammatory cytokines could lead to CKD [15, 21]. Another important mechanism of developing GVHD is invasion of inflammatory cells, such as macrophages and monocyte in various organs. Previously, mononuclear cell or T-cell infiltration

174 of renal vessels was reported to be associated with GVHD [19, 22]. GVHD or cytokines released

175 from GVHD could induce endothelial dysfunction, resulting in thrombotic microangiopathy

176 (TMA). TMA includes thrombocytopenic purpura and hemolytic uremic syndrome. Generally, 177 HSCT-induced TMA is not related to deficiencies in von Willebrand factor-cleaving protease, 178 but to endothelial dysfunction from GVHD-induced direct cell injury or by several cytokines derived from GVHD occurring in other parts of the body.

181 ABO incompatibility could induce GVHD; $A B O$ antigens are recognized in several organs

182 including endothelial cells where donor origin blood cells might attack, triggering GVHD [23,

183 24]. Thus, there is a possibility that ABO incompatibility-induced GVHD is a major risk of AKD 184 in our study rather than ABO incompatibility and GVHD respectively. 
186 disease, is one of the major complications after HSCT and could be an independent risk factor

187 for AKD development. The mechanisms of SOS-induced AKD are still unclear, but one possibility is that SOS may exacerbate liver function itself, resulting in hepatorenal syndrome. Moreover, some reports have suggested that endothelial dysfunction is essential for SOS development. We have shown that plasminogen activator inhibitor (PAI)-1 was increased in endothelial dysfunction [15]. Furthermore, previous reports indicated that the levels of PAI-1 expression could be diagnostic and predicting marker for SOS [25, 26]. In addition, use of TBI $>12$ Gy could be one of the risk factors for SOS [27]. In the present study, nine patients underwent total body irradiation with more than $12 \mathrm{~Gy}$, but development of SOS was not recognized in any patients. factor, or a disintegrin-like metalloproteinase with thrombospondin type 1 motifs 13 (ADAMTS 13 ) in our study, endothelial dysfunction might be induced by HSCTs among the AKD patients. Previous reports indicated that ABO-incompatible HSCT increased the incidence of SOS, leading to AKD $[28,29]$. Our data indicated that ABO-incompatible or GVHD-related AKD did not affect the overall survival. Similar to our results, several previous reports showed an increased mortality in ABO-incompatible transplants without an effect on overall survival [28, 29], while Kimura et al. showed lower overall survival and increased mortality rates in ABOmismatched transplants [30]. Thus, it is still unclear whether ABO-incompatible HSCT increases AKD and mortality rates.

There might be some limitations to our study, because this was a retrospective analysis and a single-center study. Several reasons could be considered. First, we could not adequately examine urine volume associated with diagnosis of AKI and we used serum creatinine as the principal criterion in the definition of AKD. Second, we did not have data on cytomegalovirus infection that often recognized in HSCT and can increase inflammation in kidney. Third, we did

211 not have precise data on severity of acute GVHD. Lastly, we did not have renal pathological data

212 that could help to enhance the understanding etiology of AKD. We would like to improve study

213 protocol including periodic urinalysis and renal biopsies.

214 


\section{CONCLUSIONS}

217

218

219

220

221

\section{2}

223

224

225

226

\section{7}

228

229

230

231

232

233

234

235

236

237

238

239

240

241

242

243

244

245

246

247

248

There is an association with ABO-incompatible HSCT and AKD as well as an association with HSCT related acute GVHD and AKD. GVHD and ABO-incompatible HSCT emerges important cause of AKD regardless nephrotoxic agents. Our data also demonstrated that mortality was not increased with the incidence of AKD.

\section{ACKNOWLEDGMENTS}

Part of this manuscript was presented at the $2^{\text {nd }}$ Asia Pacific AKI CRRT Congress 2018. We wish to thank Hitoshi Hanamoto, Hideo Yagi, and Mariko Fujita for consultation on the work-up of the patients and this study.

\section{FIGURE LEGEND}

Figure 1. Overall survival by acute kidney disease after hematopoietic stem cell transplantation.

\section{REFERENCES}

1. Klumpp, T. R. (1991) Immunohematologic complications of bone marrow transplantation, Bone marrow transplantation. 8, 159-70.

2. Sawinski, D. (2014) The kidney effects of hematopoietic stem cell transplantation, Advances in chronic kidney disease. 21, 96-105.

3. (2012) Section 2: AKI Definition, Kidney international supplements. 2, 19-36.

4. Akcan-Arikan, A., Zappitelli, M., Loftis, L. L., Washburn, K. K., Jefferson, L. S. \& Goldstein, S. L. (2007) Modified RIFLE criteria in critically ill children with acute kidney injury, Kidney international. 71, 1028-35.

5. Ricci, Z., Cruz, D. \& Ronco, C. (2008) The RIFLE criteria and mortality in acute kidney injury: A systematic review, Kidney international. 73, 538-46.

6. Mehta, R. L., Kellum, J. A., Shah, S. V., Molitoris, B. A., Ronco, C., Warnock, D. G., Levin, A. \& Acute Kidney Injury, N. (2007) Acute Kidney Injury Network: report of an initiative to improve outcomes in acute kidney injury, Critical care. 11, R31.

7. Chu, R., Li, C., Wang, S., Zou, W., Liu, G. \& Yang, L. (2014) Assessment of KDIGO definitions in patients with histopathologic evidence of acute renal disease, Clinical journal of the American Society of Nephrology : CJASN. 9, 1175-82. 
249 8. Chawla, L. S., Bellomo, R., Bihorac, A., Goldstein, S. L., Siew, E. D., Bagshaw, S. M., 250 Bittleman, D., Cruz, D., Endre, Z., Fitzgerald, R. L., Forni, L., Kane-Gill, S. L., Hoste, E., 251 Koyner, J., Liu, K. D., Macedo, E., Mehta, R., Murray, P., Nadim, M., Ostermann, M., Palevsky, 252 P. M., Pannu, N., Rosner, M., Wald, R., Zarbock, A., Ronco, C., Kellum, J. A. \& Acute Disease 253 Quality Initiative, W. (2017) Acute kidney disease and renal recovery: consensus report of the 254 Acute Disease Quality Initiative (ADQI) 16 Workgroup, Nature reviews Nephrology. 13, 241255257.

256 9. Lopes, J. A., Jorge, S. \& Neves, M. (2016) Acute kidney injury in HCT: an update, Bone 257 marrow transplantation. 51, 755-62.

258 10. Kogon, A. \& Hingorani, S. (2010) Acute kidney injury in hematopoietic cell 259 transplantation, Seminars in nephrology. 30, 615-26.

260 11. Clajus, C., Hanke, N., Gottlieb, J., Stadler, M., Weismuller, T. J., Strassburg, C. P., 261 Brocker, V., Bara, C., Lehner, F., Drube, J., Kielstein, J. T., Schwarz, A., Gueler, F., Haller, H. 262 \& Schiffer, M. (2012) Renal comorbidity after solid organ and stem cell transplantation, 263 American journal of transplantation : official journal of the American Society of Transplantation 264 and the American Society of Transplant Surgeons. 12, 1691-9.

265 12. Filipovich, A. H., Weisdorf, D., Pavletic, S., Socie, G., Wingard, J. R., Lee, S. J., Martin, P., 266 Chien, J., Przepiorka, D., Couriel, D., Cowen, E. W., Dinndorf, P., Farrell, A., Hartzman, R., 267 Henslee-Downey, J., Jacobsohn, D., McDonald, G., Mittleman, B., Rizzo, J. D., Robinson, M., 268 Schubert, M., Schultz, K., Shulman, H., Turner, M., Vogelsang, G. \& Flowers, M. E. (2005) 269 National Institutes of Health consensus development project on criteria for clinical trials in 270 chronic graft-versus-host disease: I. Diagnosis and staging working group report, Biology of 271 blood and marrow transplantation : journal of the American Society for Blood and Marrow 272 Transplantation. 11, 945-56.

273 13. Krishnappa, V., Gupta, M., Manu, G., Kwatra, S., Owusu, O. T. \& Raina, R. (2016) Acute 274 Kidney Injury in Hematopoietic Stem Cell Transplantation: A Review, International journal of 275 nephrology. 2016, 5163789.

276 14. Raeisi, S., Ghorbanihaghjo, A., Argani, H., Dastmalchi, S., Ghasemi, B., Ghazizadeh, T., 277 Rashtchizadeh, N., Nemati, M., Mesgari Abbasi, M., Bargahi, N., Samadi Kafil, H. \& Vatankhah, 278 A. M. (2017) The Effects of Valsartan on Renal Klotho Expression and Oxidative Stress in 279 Alleviation of Cyclosporine Nephrotoxicity, Transplantation.

280 15. Mima, A., Hiraoka-Yamomoto, J., Li, Q., Kitada, M., Li, C., Geraldes, P., Matsumoto, M., 281 Mizutani, K., Park, K., Cahill, C., Nishikawa, S., Rask-Madsen, C. \& King, G. L. (2012) 282 Protective effects of GLP-1 on glomerular endothelium and its inhibition by PKCbeta activation 283 in diabetes, Diabetes. 61, 2967-79.

284 16. Menzies, R. I., Howarth, A. R., Unwin, R. J., Tam, F. W., Mullins, J. J. \& Bailey, M. A. 
285 (2015) Inhibition of the purinergic P2X7 receptor improves renal perfusion in angiotensin-II286 infused rats, Kidney international. 88, 1079-87.

287 17. Pang, P., Jin, X., Proctor, B. M., Farley, M., Roy, N., Chin, M. S., von Andrian, U. H., 288 Vollmann, E., Perro, M., Hoffman, R. J., Chung, J., Chauhan, N., Mistri, M., Muslin, A. J., 289 Bonventre, J. V. \& Siedlecki, A. M. (2015) RGS4 inhibits angiotensin II signaling and 290 macrophage localization during renal reperfusion injury independent of vasospasm, Kidney 291 international. 87, 771-83.

292 18. Symington, F. W., Symington, B. E., Liu, P. Y., Viguet, H., Santhanam, U. \& Sehgal, P. B. 293 (1992) The relationship of serum IL-6 levels to acute graft-versus-host disease and hepatorenal 294 disease after human bone marrow transplantation, Transplantation. 54, 457-62.

295 19. Xun, C. Q., Thompson, J. S., Jennings, C. D. \& Brown, S. A. (1995) The effect of human 296 IL-2-activated natural killer and T cells on graft-versus-host disease and graft-versus-leukemia in 297 SCID mice bearing human leukemic cells, Transplantation. 60, 821-7.

298 20. Yang, X., Liu, C., Fujino, M., Yang, J., Li, X. K. \& Zou, H. (2017) A modified graft299 versus-host-induced model for systemic sclerosis, with pulmonary fibrosis in Rag2-deficient 300 mice, FEBS open bio. 7, 1316-1327.

301 21. Shlipak, M. G. \& Day, E. C. (2013) Biomarkers for incident CKD: a new framework for 302 interpreting the literature, Nature reviews Nephrology. 9, 478-83.

303 22. Lo, J. W., Leung, A. Y., Huang, X. R., Lie, A. K., Metz, C., Bucala, R., Liang, R. \& Lan, H. 304 Y. (2002) Macrophage migratory inhibitory factor (MIF) expression in acute graft-versus-host 305 disease (GVHD) in allogeneic hemopoietic stem cell transplant recipients, Bone marrow 306 transplantation. 30, 375-80.

307 23. Seebach, J. D., Stussi, G., Passweg, J. R., Loberiza, F. R., Jr., Gajewski, J. L., Keating, A., 308 Goerner, M., Rowlings, P. A., Tiberghien, P., Elfenbein, G. J., Gale, R. P., van Rood, J. J., 309 Reddy, V., Gluckman, E., Bolwell, B. J., Klumpp, T. R., Horowitz, M. M., Ringden, O., Barrett, 310 A. J., Blood, G. W. C. o. C. f. I. \& Marrow Transplant, R. (2005) ABO blood group barrier in 311 allogeneic bone marrow transplantation revisited, Biology of blood and marrow transplantation : 312 journal of the American Society for Blood and Marrow Transplantation. 11, 1006-13.

313 24. Stussi, G., Muntwyler, J., Passweg, J. R., Seebach, L., Schanz, U., Gmur, J., Gratwohl, A. 314 \& Seebach, J. D. (2002) Consequences of ABO incompatibility in allogeneic hematopoietic stem 315 cell transplantation, Bone marrow transplantation. 30, 87-93.

316 25. Salat, C., Holler, E., Kolb, H. J., Reinhardt, B., Pihusch, R., Wilmanns, W. \& Hiller, E. 317 (1997) Plasminogen activator inhibitor-1 confirms the diagnosis of hepatic veno-occlusive 318 disease in patients with hyperbilirubinemia after bone marrow transplantation, Blood. 89, 2184-8. 319 26. Pihusch, M., Wegner, H., Goehring, P., Salat, C., Pihusch, V., Hiller, E., Andreesen, R., 320 Kolb, H. J., Holler, E. \& Pihusch, R. (2005) Diagnosis of hepatic veno-occlusive disease by 
321 plasminogen activator inhibitor-1 plasma antigen levels: a prospective analysis in 350 allogeneic 322 hematopoietic stem cell recipients, Transplantation. 80, 1376-82.

323 27. Richardson, P. G., Ho, V. T., Giralt, S., Arai, S., Mineishi, S., Cutler, C., Antin, J. H., 324 Stavitzski, N., Niederwieser, D., Holler, E., Carreras, E. \& Soiffer, R. (2012) Safety and efficacy 325 of defibrotide for the treatment of severe hepatic veno-occlusive disease, Therapeutic advances 326 in hematology. 3, 253-65.

327 28. Benjamin, R. J. \& Antin, J. H. (1999) ABO-incompatible bone marrow transplantation: the 328 transfusion of incompatible plasma may exacerbate regimen-related toxicity, Transfusion. 39, $329 \quad 1273-4$.

330 29. Benjamin, R. J., McGurk, S., Ralston, M. S., Churchill, W. H. \& Antin, J. H. (1999) ABO 331 incompatibility as an adverse risk factor for survival after allogeneic bone marrow 332 transplantation, Transfusion. 39, 179-87.

333 30. Kimura, F., Sato, K., Kobayashi, S., Ikeda, T., Sao, H., Okamoto, S., Miyamura, K., Mori, 334 S., Akiyama, H., Hirokawa, M., Ohto, H., Ashida, H., Motoyoshi, K. \& Japan Marrow Donor, P. 335 (2008) Impact of AB0-blood group incompatibility on the outcome of recipients of bone marrow 336 transplants from unrelated donors in the Japan Marrow Donor Program, Haematologica. 93, $337 \quad 1686-93$.

338

339 
Figure 1

Overall survival by acute kidney disease after hematopoietic stem cell transplantation.

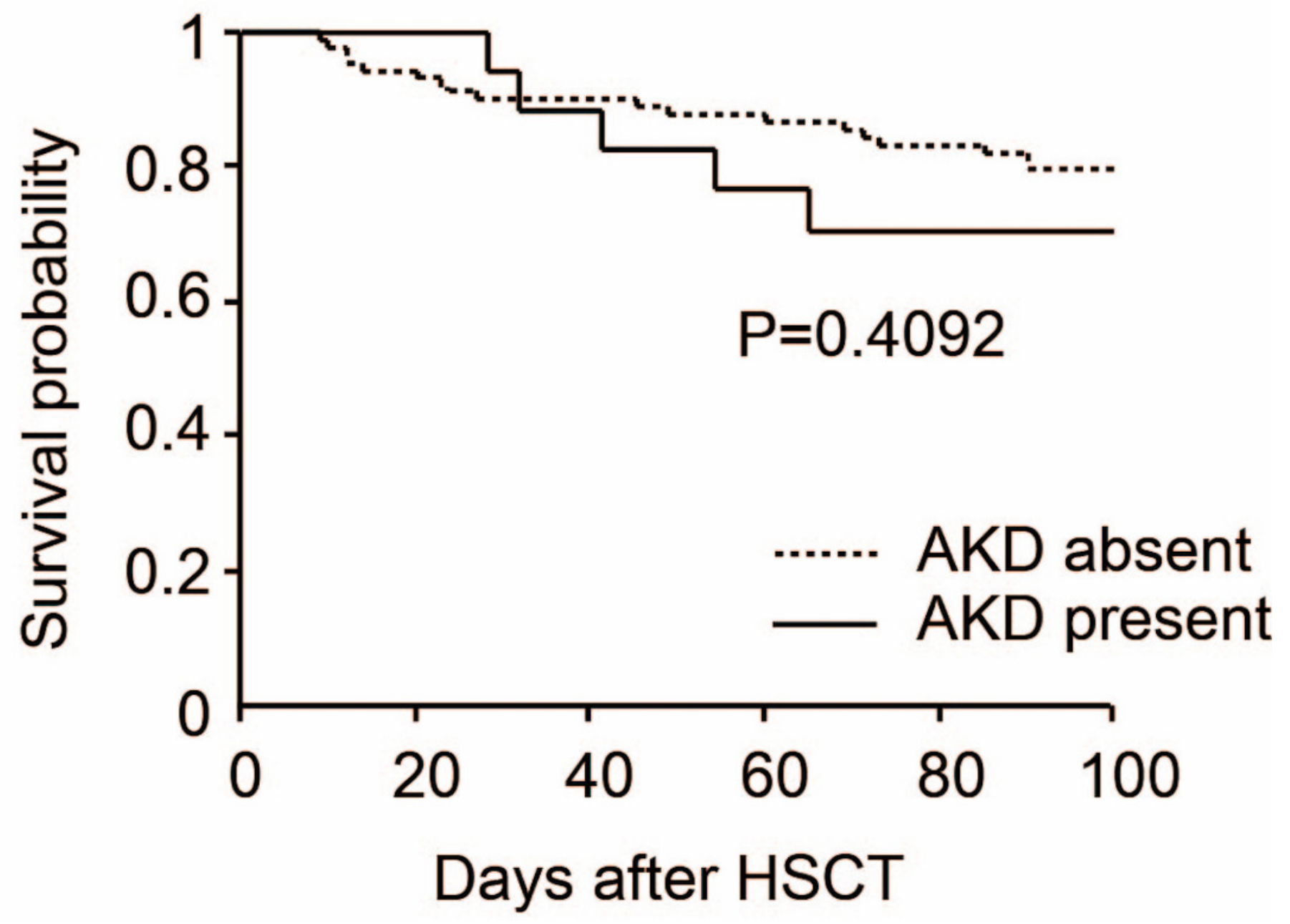




\section{Table $\mathbf{1}$ (on next page)}

Univariable association between patient characteristics and post-hematopoietic stem cell transplantation. 
Table1. Univariable association between patient characteristics and post-hematopoietic stem cell transplantation

\begin{tabular}{|c|c|c|c|c|c|}
\hline \multirow{2}{*}{\multicolumn{2}{|c|}{ Variables }} & \multirow[t]{2}{*}{ All patients $(n=108)$} & \multicolumn{2}{|c|}{ Acute kidney disease } & \multirow[t]{2}{*}{$\mathrm{P}$ value } \\
\hline & & & Absent $(n=91)$ & Present $(n=17)$ & \\
\hline Age (years) & & $49(16-70)$ & $49(17-70)$ & $49(16-66)$ & 0.574 \\
\hline Female gender & & $39(36.1 \%)$ & $34(31.5 \%)$ & $5(4.6 \%)$ & 0.594 \\
\hline Height $(\mathrm{cm})$ & & $166(149-184)$ & $166(150-184)$ & $168(149-176)$ & 0.823 \\
\hline Weight (kg) & & $58(35-95)$ & $59(35-95)$ & $53.5(43-77)$ & 0.232 \\
\hline \multicolumn{6}{|l|}{ Underlying disease } \\
\hline ALL & & $15(13.9 \%)$ & $13(12.0 \%)$ & $2(1.9 \%)$ & 0.288 \\
\hline AML & & $43(39.8 \%)$ & $37(34.2 \%)$ & $6(5.6 \%)$ & 0.592 \\
\hline CML & & $4(3.7 \%)$ & $4(3.7 \%)$ & $0(0 \%)$ & 0.999 \\
\hline MDS & & $10(9.3 \%)$ & $7(6.5 \%)$ & $3(2.8 \%)$ & 0.371 \\
\hline MM & & $5(4.6 \%)$ & $5(4.6 \%)$ & $0(0 \%)$ & 0.999 \\
\hline AA & & $6(5.6 \%)$ & $4(3.7 \%)$ & $2(1.9 \%)$ & 0.265 \\
\hline Others & & $25(23.1 \%)$ & $24(22.2 \%)$ & $1(0.9 \%)$ & 0.063 \\
\hline $\mathrm{HTN}$ & & $12(11.1 \%)$ & $2(1.9 \%)$ & $10(9.3 \%)$ & 0.999 \\
\hline DM & & $9(8.3 \%)$ & $7(6.5 \%)$ & $2(1.9 \%)$ & 0.628 \\
\hline $\mathrm{TBI} \geq 8 \mathrm{~Gy}$ & & $79(73.1 \%)$ & $67(62.0 \%)$ & $12(11.1 \%)$ & 0.726 \\
\hline ABO incompatible & & $18(16.7 \%)$ & $10(9.3 \%)$ & $8(7.4 \%)$ & 0.001 \\
\hline Acute GVHD & & $17(15.7 \%)$ & $7(6.5 \%)$ & $10(9.3 \%)$ & $<0.001$ \\
\hline $\begin{array}{l}\text { CNI-based } \\
\text { prophylaxis }\end{array}$ & GVHD & $67(62.0 \%)$ & $56(51.2 \%)$ & $11(0.2 \%)$ & 0.999 \\
\hline \multicolumn{6}{|c|}{ Donor and cell source } \\
\hline Allo-BMT & & $20(18.5 \%)$ & $17(15.7 \%)$ & $3(2.8 \%)$ & 0.999 \\
\hline UR-CBT & & $38(35.2 \%)$ & $31(28.7 \%)$ & $7(6.5 \%)$ & 0.589 \\
\hline
\end{tabular}




\begin{tabular}{lllll} 
UR-BMT & $26(24.1 \%)$ & $21(19.4 \%)$ & $5(4.6 \%)$ & 0.550 \\
Allo-PBSCT & $11(10.2 \%)$ & $10(9.3 \%)$ & $1(0.9 \%)$ & 0.999 \\
Auto-PBSCT & $16(14.8 \%)$ & $15(13.9 \%)$ & $1(0.9 \%)$ & 0.458 \\
\hline
\end{tabular}

2 ALL: acute lymphoblastic leukemia, AML: acute myeloblastic leukemia, CML: chronic myeloblastic leukemia, MDS: 3 myelodysplastic syndromes, MM: multiple myeloma, AA: aplastic anemia, HTN: hypertension, DM: diabetes mellitus, TBI: total 4 body irradiation, GVHD: graft versus host disease, CNI: calcineurin inhibitor, Allo: allogenic, BMT: bone marrow transplantation, 5 UR: unrelated, CBT: umbilical cord blood transplantation,

6 PBSCT: peripheral blood stem cell transplantation, Auto: autologous 